\title{
NONLINEARITY AND FORECASTING ASPECTS OF PERIODICALLY INTEGRATED AUTOREGRESSIONS
}

\author{
by
}

\author{
PHILIP HANS FRANSES
}

Econometric Institute, Erasmus University Rotterdan P.O. Box 1738, NL-3000 DR Rotterdam, The Netherlands

\section{Summary}

This paper deals with forecasting and nonlinearity aspects of linear periodic models for seasonally observed time series which contain a single unit root. This unit root imposes a nonlinear restriction on the model parameters. Multi-step ahead forecasts differ from forecasts obtained from nonperiodic models in the sense that they can reflect slowly changing seasonal patterns observed within the estimation sample.

Keywords Seasonal time series, periodic models, unit roots

\section{Introduction}

Two often encountered characteristics of seasonally observed time series in economics is that they show trending behavior and slowly changing seasonal patterns. Traditionally, time series analysts describe such variables using linear autoregressive models for the appropriately transformed series. Usually these transformations are based on the application of the first order differencing filter or the seasonal differencing filter. These filters imply for log transformed time series that the resulting series correspond to seasonal and annual growth rates, respectively.

For some economic series these models can be considered to be only rough approximations. Seasonal growth rate models assume that the seasonal patterns can be described by constant seasonal dummies. However, recursive estimates of the corresponding parameters sometimes suggest that they may not be constant within the estimation sample, see, e.g., Canova and Ghysels (1992). On the other hand, the annual growth rate models assume that the annual series, which contain the observations in the separate seasons, each have a unit root. In theory, this implies that the observations in the different seasons may not have the tendency to move together, which may be unreasonable for many economic time series. This calls for a class of models that can cope with these drawbacks of the traditional models. An example of such a class is that of periodic autoregressions with a single unit root, which are also called periodically integrated autoregressions [PIAR]. Detailed discussions of these models are given in Osborn (1988), Franses (1991), and Boswijk and Franses (1992). In the present paper the focus is on the forecasting and nonlinearity aspects of this class.

First, in section 2, some notational issues are briefly discussed. Then, in section 3, the nonlinearity aspect is highlighted. Section 4 deals with forecasts from a PIAR. Section 5 concludes with some remarks. 


\section{Preliminaries}

Consider a time series $x$, which is observed $s$ times per year during $N$ years, where $s$ usually is 2, 4 or 12 . The index $t$ runs from 1 through $n$, with $n=s N$. A commonly applied assumption for $x_{t}$ is that it can be described by an autoregression of order $p$ $[\operatorname{AR}(p)]$, i.e.

$$
\phi_{p}(B) x_{t}=\mu+\varepsilon_{t},
$$

where $\phi_{p}(B)=1-\phi_{1} B-\ldots-\phi_{p} B^{p}$ is a $p$-th order polynomial in the operator $B$ which is defined by $B^{k} x_{t}=x_{t-k}$. The $\varepsilon_{t}$ is a standard white noise process, and $\mu$ is a constant.

When $\phi_{p}(B)$ can be decomposed as $\phi_{p}(B)=\phi_{p-s}^{*}(B)\left(1-B^{s}\right)$ model $(1)$ becomes $\phi_{n-s}^{*}(B) y_{t}$ $=\mu+\varepsilon_{i}$, where $y_{t}=\Delta_{s} x_{t}$ i.e., the annually differenced $x_{t}$ series. The $\Delta_{s}$ filter is defined by $\Delta_{j} x_{t}=x_{t}-x_{i-j}$. Alternatively, when $\phi_{p}(B)$ in $(1)$ can be decomposed as $\phi_{p-1}^{* *}(B)(1-B)$, then model (1) becomes $\phi_{p-1}^{* *}(B) z_{l}=\mu+\varepsilon_{t}$, where $z_{l}=\Delta_{1} x_{t}$ with $\Delta_{1}$ is the first order differencing filter. In the latter case, the $\mu$ is usually replaced by seasonal dummies to account for seasonal variation.

Model (1) assumes autoregressive parameter constancy, i.e. the $\phi_{i}$ parameters do not vary with season $s$. A model that allows periodic parameters is the so-called periodic autoregression of order $p[\operatorname{PAR}(p)]$, see, e.g., Anderson and Vecchia (1993) and the references cited therein. There are several ways to represent a PAR process, of which a simple one is

$$
\phi_{p s}(B) x_{t}=\mu_{s}+\varepsilon_{t},
$$

with $\phi_{p s}(B)=1-\phi_{1 s} B-\ldots-\phi_{p s} B^{p}$, where the index $s$ indicates that the parameters vary with the season. Of course not all parameters in $\phi_{p s}(B)$ have to be unequal to zero. Hence, also the lag lengths in each of the seasons may be different.

It is clear from (2) that a PAR model considers each season differently. Therefore, an alternative representation of (2) is given by a multivariate model for $X_{T}$ which is the ( $\left.s \times 1\right)$ vector of stacked seasonal observations, i.e. $X_{T}=\left(X_{1 T}, X_{2 T}, \ldots, X_{s T}\right)^{\prime}$, where $X_{i T}$ is the observation in season $i$ in year $T$, see, e.g., Lutkepohl (1991) and the references cited therein. The index $T$ runs from 1 through $N$. A vector representation of (2) is

$$
A_{0} X_{T}=\delta+A_{1} X_{T-1}+\ldots+A_{m} X_{T-m}+\varepsilon_{T}
$$

where $A_{i}, i=0, \ldots, m$ are (sxs) parameter matrices, $\delta$ is the (sx1) vector of constants, and $\mathbf{E}_{T}$ is vector white noise process. The order $m$ is related to the order $p$ in (2) by $m \leq s p$.

\section{A Unit Root and Nonlinearity}

The vector process $X_{T}$ and the univariate process $x_{t}$ do not contain unit roots if the solutions to the characteristic equation 


$$
\left|A_{0}-A_{1} z-\ldots-A_{m} z^{m}\right|=0
$$

are outside the unit circle. Franses and Paap (1993) in a yet unpublished paper find evidence of the presence of a single unit root in about twenty quarterly UK macroeconomic time series. The presence of a unit root can be checked by testing whether $z=l$ is the only unity solution of (4). It is clear from (1) that this unity solution implies a nonlinear parameter restriction. Hence, a periodically integrated autoregression is a linear model with a nonlinear parameter restriction. A simple example is given by a PAR(1) process

$$
x_{1}=\phi_{1 s} x_{1-1}+\varepsilon_{1} .
$$

The multivariate model for the corresponding $X_{T}$ vector process contains a unit root when $\Pi_{i=1}^{s} \phi_{1 i}=1$.

Pre-multiplying (3) with $A_{0}{ }^{-1}$, and some rewriting yields

$$
\Delta X_{T}=\delta+\Gamma_{1} \Delta X_{T-1}+\ldots+\Gamma_{m-1} \Delta X_{T-m+1}+\Pi \Pi X_{t-m}+\omega_{T}
$$

where the $\Gamma_{j}$ and $\Pi, j=1, \ldots, m-1$, are functions of the $A_{i}$ in (3), $i=0, \ldots, m$, where $\Delta$ is the first order differencing filter for annual time series, and $\omega_{T}=A_{0}{ }^{-1} \varepsilon_{T}$. Note that the $\Delta$ filter here corresponds to the $\Delta_{s}$ filter for the univariate quarterly series $X_{i}$.

The representation in (5) is convenient to test for cointegration between the elements of $X_{T}$, see Franses (1991). For example, if the rank of the matrix $\Pi$ is equal to zero, the model reduces to a vector process for the $\Delta X_{T}$ series. Otherwise stated, when there are no cointegration relations between the elements of $X_{T}$, the transformed series $\Delta_{S} X$, can be used for further modeling. Then, each of the time series $X_{s T}$ contains a stochastic trend, and there are no linear combinations that ensure that these series tie together.

A PIAR process of some order $p$ which contains a single unit root has $s-I$ cointegrating relations between the $X_{s T}$ series. Hence, the rank of $\Pi$ in (5) is equal to $s-1$. If this is the case, it can easily be shown that these cointegration relations are given by

$$
X_{i T}-\alpha_{i} X_{i-1, T}, \text { with } \prod_{j=1}^{s} \alpha_{j}=1 \text { for } i=1,2, \ldots, s
$$

where $X_{0 T}=X_{s, T-1}$. Hence, a $\operatorname{PIAR}(p)$ process can also be written as

$$
\left(1-\alpha_{i} B\right) x_{t}=\beta_{1 s}\left(1-\alpha_{i-1} B\right) x_{t-1}+\ldots+\beta_{p-1, s}\left(1-\alpha_{i-p+1} B\right) x_{t-p+1}+\mu_{s}+\varepsilon_{1}
$$

where the $\alpha_{i}$ satisfy the restriction in (6), and where $\alpha_{-k}=\alpha_{s-k}$, for $k=0,1, \ldots$. The $\beta_{j s}$ are again periodic parameters. The filter $\left(1-\alpha_{i} B\right)$ is called a periodic differencing filter. Obviously, when all $\alpha_{i}$ are equal to one, the conventional $(1-B)$ filter emerges.

Together with (4), the equation in (7) indicates a useful model selection strategy. A first step is to estimate the order $p$ of the PAR. This can be done using familiar model 
selection criteria as the Akaike and Schwarz information criteria, or using $F$ type tests for parameter redundancy. Once this order is determined, the next step is to test whether the autoregressive parameters are seasonally varying. Again an $F$ type test can be constructed. In Franses and Paap (1993) it is shown that for both $F$ tests standard asymptotic results apply. The third step is to check the number of unit roots in a PAR by checking the solutions of (4). When there is only a single unit root, which is usually the case in practice, one can reformulate the model as in (7) to test for adequacy of filters such as ( $I$ $B)$, or even $\left(1-B^{4}\right)$ if the order $p$ is large enough.

The model selection strategy, discussed in the previous paragraph, is a general-tosimple-method. The crucial tests for periodicity and unit roots are performed within the context of a prespecified model. An alternative, since simple-to-general, method is given in Vecchia and Ballerini (1991). This approach checks for periodicity in the autocorrelation function. A drawback of this method however is that two distinct time series processes, like $\operatorname{AR}(2)$ and $A R(3)$, can have similar estimated autocorrelation functions. Moreover, not rejecting the null hypothesis of no periodic autocorrelations does not automatically imply that the underlying process is nonperiodic.

\section{Forecasting}

Consider again the expressions in (6) and (7). In practice, the $\alpha_{i}$ are usually estimated to be close to, though not equal to, one. This means that the distance between the observations $X_{i T}$ and $X_{i-1, T}$ is not constant over time, which implies that a PIAR process displays a slowly changing seasonal pattern. The changes in this pattern depend on the changes in the stochastic trend in the $X_{T}$ process.

The cointegration relations in (6) also effect the pattern of the multistep ahead forecasts. In fact, these forecasts will display slowly changing patterns too. The magnitudes of these changes depend on the estimated values of $\alpha_{i}$. On the other hand, the nonperiodic models for the $\Delta_{4}$ and $\Delta_{1}$ transformed time series do not generate such slowly changing patterns in the forecasts.

Of course, when the $(1-\alpha, B)$ filter is appropriate, one can expect some gain in onestep ahead forecasting of using PIAR models. This is because some nonperiodic models misspecify either the number or the form of the cointegration relations. Empirical evidence of such a gain is reported in Osborn and Smith (1989), Franses (1992), and Franses and Romijn (1992).

\section{Remarks}

Periodically integrated autoregressions can be useful for the description of time series with a stochastic trend and a slowly changing seasonal pattem. This seasonal pattern changes because of variations in the stochastic trend. In other words, seasonality, trend and possibly cycles may not be easily separable. Since this is the underlying assumption of seasonal adjustment methods as Census-X11, one may question the usefulness of these methods when applied to periodic time series with a single unit root. Future research will be directed to investigate the effects of seasonal correction methods.

Although Osborn (1988) derives a PIAR(1) process for nondurable quarterly consumption directly from an economic theory, for many economic time series it is unlikely that a univariate PIAR process is the underlying data generating process. A natural step may then 
be to consider periodic cointegration models. These models incorporate error correction mechanisms with periodic equilibrium and adjustment parameters.

\section{Acknowledgements}

Thanks are due to an anonymous referee for several constructive comments. The financial support from the Royal Netherlands Academy of Arts and Sciences is gratefully acknowledged.

\section{References}

Anderson, P.L. and A.V. Vechhia (1993), 'Asymptotic results for periodic autoregressive moving-average processes', J. of Time Series Analysis, 14, 1-18.

Boswijk, H.P. and P.H. Franses (1992), 'Testing for periodic integration', Econometric Institute Report 9216, Erasmus University Rotterdam.

Canova, F. and E. Ghysels (1991), 'Changes in seasonal patterns: are they cyclical?', J. of Economic Dynamics and Control, to appear.

Franses, P.H. (1991), 'A multivariate approach to modeling univariate seasonal time series', Econometric Institute Report 9101, J. of Econometrics, to appear.

Franses, P.H. (1992), 'Periodically integrated subset autoregressions for Dutch industrial production and money stock', $J$. of Forecasting, to appear.

Franses, P.H. and R. Paap (1993), 'Model selection in periodic autoregressions', Econometric Institute Report 9213, Erasmus University Rotterdam.

Franses, P.H. and G. Romijn (1992), 'Periodic integration in UK macroeconomic time series', Econometric Institute Report 9209, Int. J. of Forecasting, to appear.

Lïtkepohl, H. (1991), Introduction to multiple time series analysis, Berlin: Springer Verlag.

Osborn, D.R. (1988), 'Seasonality and habit persistence in a life-cycle model of consumption', J. of Applied Econometrics, 3, 255-266.

Osborn, D.R. and J.P. Smith (1989), 'The performance of periodic autoregressive models in forecasting seasonal U.K. consumption', $J$. of Business and Economic Statistics, 7, 117-127.

Vecchia, A.V. and R. Ballerini (1991), 'Testing for periodic autocorrelations in seasonal time series data', Biometrika, 78, 53-63. 